\title{
Warburg Effect
}

National Cancer Institute

\section{Source}

National Cancer Institute. Warburg Effect. NCI Thesaurus. Code C19820.

This effect refers to the high cellular levels of lactic acid in tumor cells, and is named after Otto Warburg who worked on photosynthesis. Tumor cells use excessive glucose in their rapid growth, and lactic acid is a product of the glycolytic pathway. This process is not inhibited by oxygen, therefore, it is also called aerobic glycolysis. 